\title{
The frequency of the known mitochondrial variants associated with drug-induced toxicity in a Korean population
}

Vinh Hoa Pham ${ }^{1}$, Van Lam Nguyen ${ }^{1}$, Hye-Eun Jung ${ }^{1,2}$, Yong-Soon Cho ${ }^{1,3,4}$ and Jae-Gook Shin ${ }^{1,3,4^{*}}$

\begin{abstract}
Background: Few studies have annotated the whole mitochondrial DNA (mtDNA) genome associated with drug responses in Asian populations. This study aimed to characterize mtDNA genetic profiles, especially the distribution and frequency of well-known genetic biomarkers associated with diseases and drug-induced toxicity in a Korean population.

Method: Whole mitochondrial genome was sequenced for 118 Korean subjects by using a next-generation sequencing approach. The bioinformatic pipeline was constructed for variant calling, haplogroup classification and annotation of mitochondrial mutation.

Results: A total of 681 variants was identified among all subjects. The MT-TRNP gene and displacement loop showed the highest numbers of variants (113 and 74 variants, respectively). The m.16189T >C allele, which is known to reduce the mtDNA copy number in human cells was detected in $25.4 \%$ of subjects. The variants (m.2706A> G, m.3010A> G, and $\mathrm{m} .1095 \mathrm{~T}>\mathrm{C}$ ), which are associated with drug-induced toxicity, were observed with the frequency of $99.15 \%$, $30.51 \%$, and $0.08 \%$, respectively. The $\mathrm{m} .2150 \mathrm{~T}>\mathrm{A}$, a genotype associated with highly disruptive effects on mitochondrial ribosomes, was identified in five subjects. The $\mathrm{D}$ and $\mathrm{M}$ groups were the most dominant groups with the frequency of $34.74 \%$ and $16.1 \%$, respectively.

Conclusions: Our finding was consistent with Korean Genome Project and well reflected the unique profile of mitochondrial haplogroup distribution. It was the first study to annotate the whole mitochondrial genome with drug-induced toxicity to predict the ADRs event in clinical implementation for Korean subjects. This approach could be extended for further study for validation of the potential ethnic-specific mitochondrial genetic biomarkers in the Korean population.
\end{abstract}

Keywords: Mitochondria, Polymorphism, Drug-induced toxicity, Korean population

\section{Background}

Mitochondria are organelles that play a central role in cellular energy suppliers. They have their own genome and genetic code, and an exceptionally high mutation rate

\footnotetext{
*Correspondence: phshinjg@gmail.com

${ }^{1}$ Department of Pharmacology and Pharmacogenomics Research Center,

Inje University, College of Medicine, 633-165 Gaegum-Dong, Jin-Gu,

Busan, Republic of Korea

Full list of author information is available at the end of the article
}

[1]. At least 100 mutations are expected to be observed in almost $90 \%$ of non-proliferating cells, while no other cell types have fewer than 10 mutations by the age of 70 years [2]. Polymorphism of mitochondrial DNA (mtDNA) is associated with various pathophysiology, and could explain diverse vulnerability to the diseases or drug toxicity [3]. The high variability of human mitochondria has been studied in the context of common diseases. Ninetyfive mitochondrial markers in the Mitomap database 
have been confirmed to be pathogenic as of October 2021 [4]. Pathogenic mtDNA mutations were reported to be common in the general population, and are also present in some major haplogroups. Therefore, many healthy individuals carry potential harmful variants. At least 1 in every 200 healthy subjects harbours a pathogenic variant that can be a potential cause of disease in the next generation [5].

In addition, mitochondrial polymorphisms were reported to be associated with drug responses, and can reveal the role of mtDNA variation in susceptibility to drug toxicity. It has been reported that variations in mtDNA can result in differences in mitochondrial function that, in turn, may lead to idiosyncratic drug-induced toxicity [6]. Limited information, however, is available about the roles of mitochondrial haplogroups in susceptibility to drug-induced toxicity. Anti-retroviral therapy (ART), antibiotics and chemotherapeutic agents are well known drug classes in relation to the mitochondria mediated drug toxicities [7]. The mtDNA mutations and copy numbers have been proposed as potential biomarkers to monitor therapeutic responses and prognosis in cancer treatments, although the mechanisms are not well established $[8,9]$. There are reports that specific mtDNA haplogroups are associated with ART-induced peripheral neuropathy and metabolic disorders from clinical studies conducted in European patients [10-15]. The susceptibility of antibiotics toxicity is also well known to associated with mtDNA polymorphisms, especially in mitochondrial $12 \mathrm{~S}$ rRNA and $16 \mathrm{~S}$ rRNA genes which play an important role in preventing or inducing toxicity [16].

The mtDNA has a unique genetic feature known as 'heteroplasmy', which allows mutant and wild-type mtDNAs to coexist. It is well known that healthy individuals harbour relatively low levels $(<1 \%)$ of mtDNA heteroplasmy in general. It is known that the mtDNA heteroplasmy increases throughout the lifespan, and the initial mutant mtDNA will be the predominant type within a specific cell. Once a certain threshold level of mutant mtDNA is above the normal range, the mutant load influences on the cellular and patient phenotypes. Hence, a low level of heteroplasmy would be linked to late-onset of related diseases in an individual subject [17]. Since the Next-generation sequencing (NGS) technology is a tool to identify and quantify the level of heteroplasmy at the $1 \%$ range, this high throughput technology has been implemented extensively towards decoding the mitochondrial genome in many ethnic populations [18-22]. Two reports are available so far for the NGS-based mitochondrial genome study in the Korean subjects [23, 24], and they found the similar profile of mitochondrial genetic variants to that in the Japanese and Chinese Han populations [19, 25]. However, those reports are not issued on the potential implementation of those mtDNA variants as potential biomarkers for the prediction of drug induced toxicity.

In this study, therefore, the mtDNA genetic profiles of 118 Korean subjects were analysed by using the NGS approach, in order to investigate the distributions and frequencies of known mtDNA variant biomarkers associated with diseases and drug-induced toxicity in a Korean population.

\section{Methods}

Sample collection and DNA extraction

DNA samples were harvested from peripheral blood that were selected from biobank in Pharmacogenomics Research Center, Inje University, Korea. In totally, 118 independent Korean subjects, all of whom provided written informed consent to participate in the genotyping analysis, were adults with a mean age of 37.1 years (range 19-65 years), and a mean BMI of 22.1 (range $15.1-34.1 \mathrm{~kg} \mathrm{~m}^{-2}$ ). The study population consisted of 65 males (55\%) and 88 (74.6\%) non-smokers. Sixty-eight subjects were collected from multicentre for Personalised Precision Medicine of Tuberculosis from 2018 to December 2020. The subjects with age from 18 to 65 years old, were diagnosed with drug-susceptible tuberculosis and treated with first-line anti-tuberculosis drugs (rifamycin, isoniazid, pyrazinamide and ethambutol). The subjects had no adverse drug reactions, no comorbidities and normal laboratory test results during treatment. The remaining 50 individuals were selected from 1000 Korean healthy subjects that were recruited from September 1, 2005 to August 31, 2015 with the support of Ministry of Health and Welfare, Korea for establishment of a pharmacogenetics database for the healthy Korean adults. The selected subjects were individuals over 19 years old without any recorded diseases at the time of sample collection. The characteristic of subjects in this study were described as in Additional file 1: Table S1.

Blood samples were collected at the time of recruitment and frozen at $-80^{\circ} \mathrm{C}$. Total DNA was isolated from peripheral blood samples using a QIAamp DNA Blood Mini Kit (Qiagen, German) according to the manufacturer's instructions. DNA purity and concentration were measured using a NanoDrop 2000 Spectrophotometer (Thermo Fisher Scientific, USA).

\section{Amplification of mitochondrial long-range fragments}

A well-established method for exclusive amplification of mitochondrial long-range fragments as described by Gould et al. [26] was used to avoid the co-amplification of nuclear genomic DNA. The PCR primers designed for the study included MT-L1_F (5'-AAATCTTACCCC GCCTGTTT-3), MT-L1_R (5'-AATTAGGCTGTG 
GGTGGTTG-3), MT-L2_F (5'-GCCATACTAGTC TTTGCCGC- 3), and MT-L2_R (5'-GGCAGGTCA ATTTCACTGGT-3') to amplify entire sequence of a mitochondrial genome. We used an Applied Biosystems (GeneAmp PCR system 9700, Thermo Fisher Scientific, USA) for analysing long-range fragments 1 and 2 (MTL1 and MT-L2, respectively) of mitochondrial genomes ( $~ 8.5 \mathrm{~kb}$ each), as described by McElhoe et al. The PCR was performed using 50- $\mu$ l reaction mixtures containing $200 \mathrm{ng}$ of genomic DNA with LA Taq ${ }^{\circledR}$ and $2 \times \mathrm{GC}$ buffer (TaKaRa, Japan) [27]. The PCR conditions for both the MT-L1 and MT-L2 fragments consisted of an initial denaturation step at $94{ }^{\circ} \mathrm{C}$ for 1 min followed by 30 cycles of denaturation at $94{ }^{\circ} \mathrm{C}$ for $30 \mathrm{~s}$, annealing at $59{ }^{\circ} \mathrm{C}$ for $40 \mathrm{~s}$, extension at $72{ }^{\circ} \mathrm{C}$ for $9 \mathrm{~min}$, and a final extension step of $72{ }^{\circ} \mathrm{C}$ for $10 \mathrm{~min}$. The DNA template (9947A) from the National Institute of Standards and Technology (NIST, USA) [28] was utilised as the mitochondrial standard DNA for sequencing.

\section{Mitochondrial whole-genome sequencing}

DNA libraries with an expected insert size of $\sim 200 \mathrm{bp}$ were prepared using NEBNext (UK) in accordance with the instruction manual provided [29], and optimised for our samples. The concentrations of mitochondrial libraries were measured using the QuantiFluor ${ }^{\circledR}$ ONE dsDNA system (Promega, USA). Equimolar amounts of the 60 indexed libraries were pooled to obtain a 4-nM mixture. After denaturation and further dilution, the final 16 pM of this mixture was loaded into an Illumina cartridge. Sequencing was performed using the Illumina MiSeq Reagent kit v2 (300 cycles) and MiSeqDx instrument (Illumina, USA) in accordance with the manufacturer's instructions.

\section{Analysis of mitochondrial genomes}

A bioinformatics pipeline was developed to reconstruct and analyse human mtDNA from high-throughput sequencing data as shown in Fig. 1. The sequencing data obtained from the Illumina MiSeqDx were quality-controlled using FastQC (version 0.11.9) [30] and MultiQC (version 1.10.1) [31]. All adapters and sequences with a quality score $<30$ and sequence length $<70$ bp were removed using Trimmomatic (version 0.39) [32]. In the alignment process, to minimise the nuclear mitochondrial DNA segment (NUMT), the qualified data of each sample were aligned simultaneously to human genome reference hg19 based on the revised Cambridge Reference Sequence (rCRS) of human mitochondrial DNA [33] using BWA (version 0.7.17-r1188) [34]. Regarding downstream alignment processes such as conversion from Sequence Alignment/Map (SAM) to Binary Alignment/Map (BAM) format and marking and removing duplicates were performed using GATK (version 4.1.8.1). The final alignment assessment was performed using Qualimap (version v.2.2.1) [35]. Variants and heteroplasmy were called using GATK Mutect2 (version 4.1.8.1) in mitochondrial mode. All variants were filtered for a heteroplasmy level $<5 \%$. Other filtering processes were in accordance with the instructions of the GATK best practices for mitochondrial genomes [36]. The results of the variant-calling pipeline were confirmed based on the NIST mtDNA standard (GM09947A) [28] before applying to real samples. Finally, all variant-calling pipelines were combined. The variants were annotated using HmtNote (version 0.7.2) [37] against the HmtVar database [38]. The haplogroups and sub-haplogroups of all samples in the cohort were defined based on PhyloTree 17 nomenclature that is integrated in Haplogroup2 [39].

\section{Results \\ Distribution of mitochondrial variants in the sequenced mtDNAs}

The bioinformatics pipeline was confirmed to be consistent with the variant-calling pipeline for the mtDNA standard sequence [28]. According to NIST [40], the percentage of heteroplasmy for m.1393G > A was $17.4 \pm 1.7 \%$ and that for $\mathrm{m} .7861 \mathrm{~T}>\mathrm{C}$ was $74.6 \pm 14.5 \%$, while the values in our study were $17.4 \pm 0.6 \%$ and $89.5 \pm 0.1 \%$, respectively. These results confirmed the accuracy of the bioinformatics pipeline and experimental procedure for mtDNA sequencing and analysis. The average mtDNA coverage for the examined samples was $2601 \mathrm{X} \pm 685 \mathrm{X}$ (detailed information of average coverage for each sample was shown in Additional file 1: Figure S1). The average read length for all paired sequences was $203 \pm 15 \mathrm{bp}$.

We identified a total of 681 variants called from 118 individuals (Fig. 2). Most of the variable positions were transitions $(86.5 \%$ overall), while the overall percentage of transversions was about 3\%. Our study showed that transitions were dominant, as reported previously for human mitochondrial genomes [41]. Indel polymorphism was detected at a rate of $8.5 \%$ among all individuals. The median of the mtDNA variants was 38 (range 26-55). No variants were found in 9/22 tRNA-coding genes. $M T$ TRNP gene and the displacement loop (D-loop) had the most polymorphisms (113 and 74 variants, respectively) (Additional file 1: Table S2). No disease mutations with 'confirmed' status in the current MITOMAP database [4] were found in any individuals.

The lowest heteroplasmy level of mtDNA variants was $10 \%$ for several non-coding variants. The levels of heteroplasmy of some mutations (m.3206C $>\mathrm{T}$, m.4824A $>$ G, m.8473T $>C, \quad$ m.16179CA $>C$, and m.16183A > C) varied from approximately $10 \%$ to $100 \%$ 


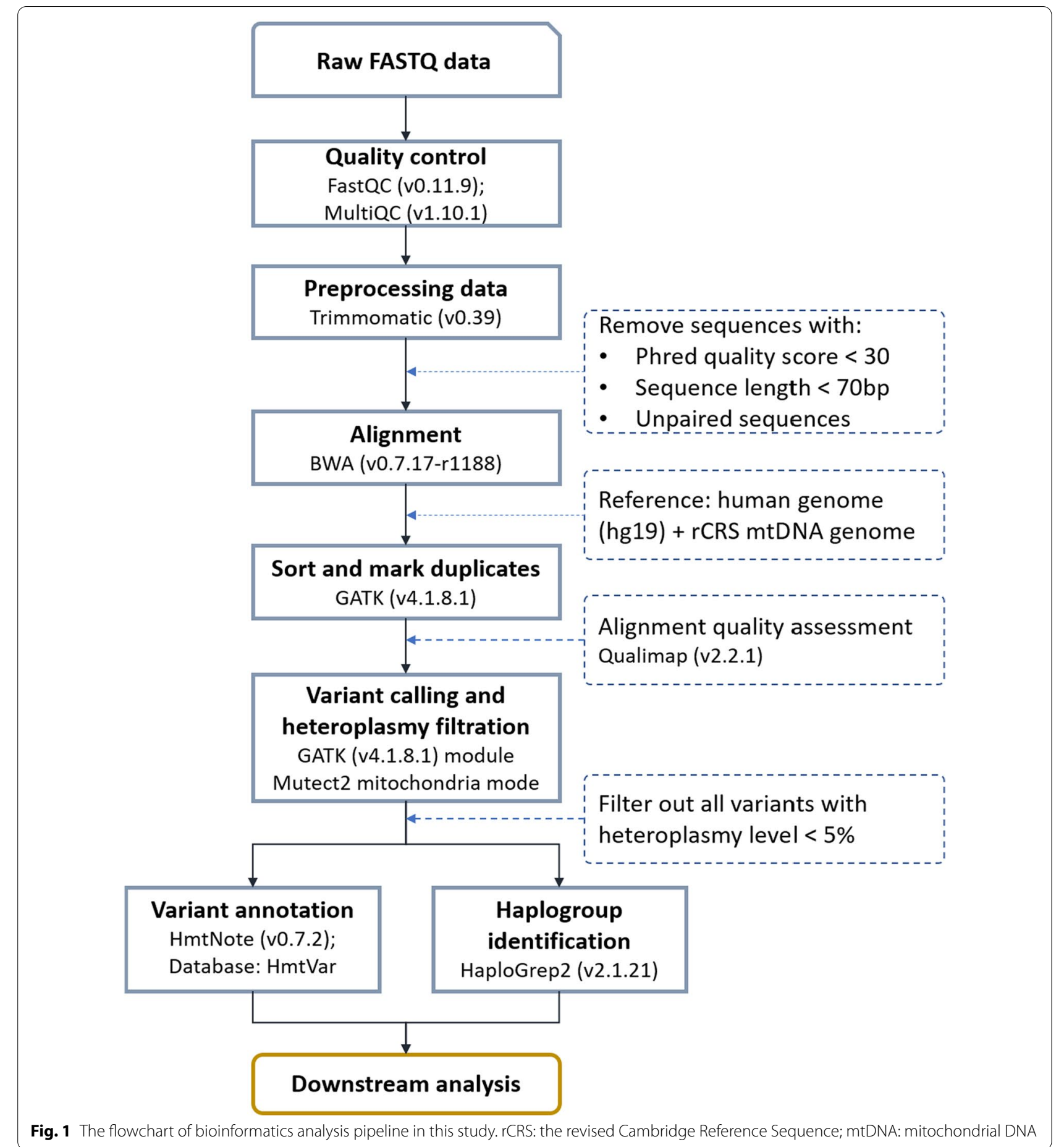

Fig. 1 The flowchart of bioinformatics analysis pipeline in this study. rCRS: the revised Cambridge Reference Sequence; mtDNA: mitochondrial DNA

(homoplasmy) and their allele frequency in the studied population was $11 \%$. The $\mathrm{m} .16189 \mathrm{~T}>\mathrm{C}$ polymorphism causing a lower mtDNA copy number was present in
$29 / 118(24.58 \%)$ samples as homoplasmy, and in one healthy individual at a heteroplasmy level of $54.6 \%$ (Additional file 1: Table S3). 


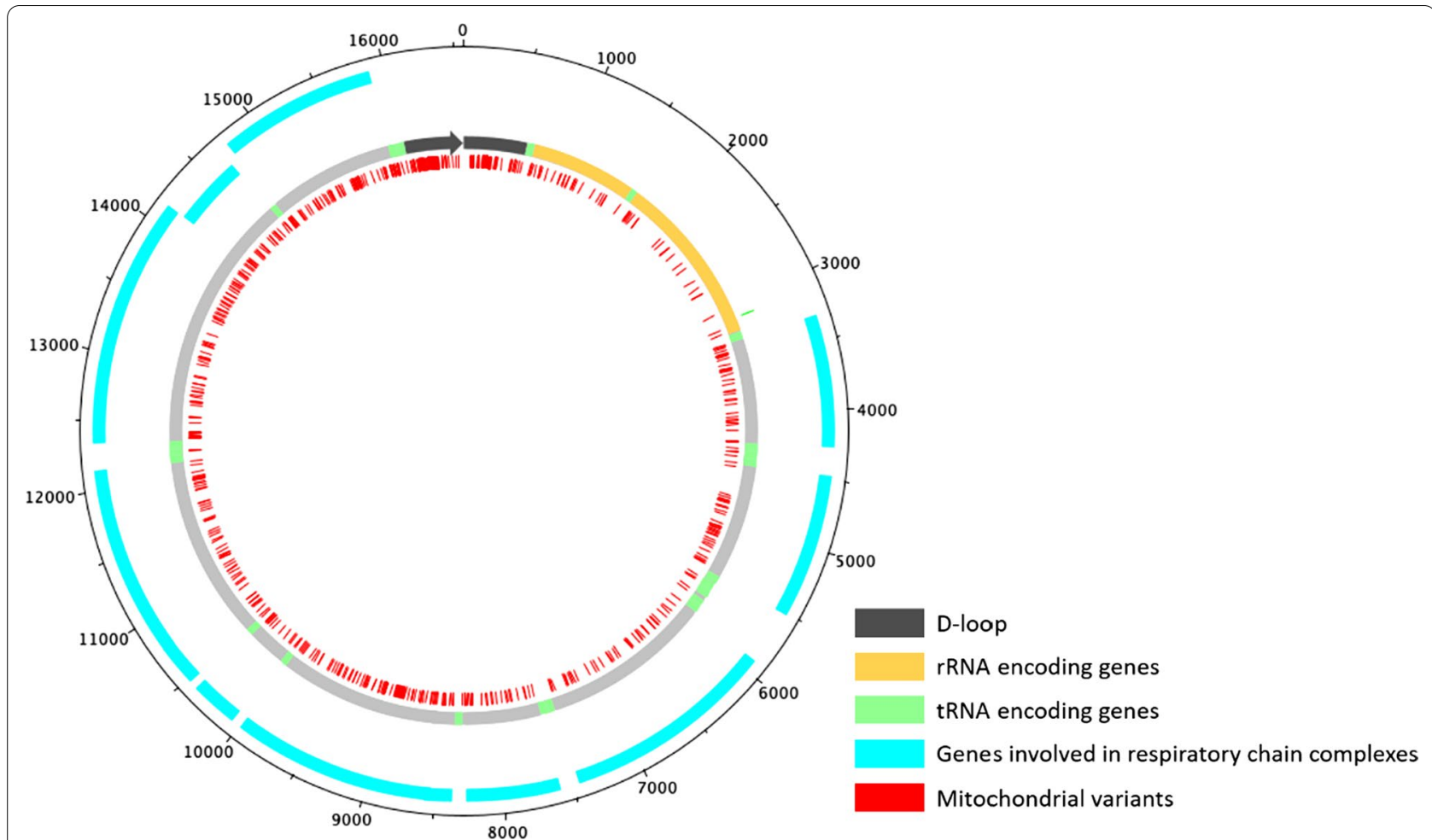

Fig. 2 Distribution of variants in mitochondrial genomes in this study. Mitochondrial variants (red) in tRNA genes (green), rRNA genes (orange) and genes involved in mitochondrial respiratory chain complexes (blue), in the Korean population in the present study $(n=118)$ according to the Revised Cambridge Reference Sequence (NC_012920.1). The mitochondrial genome was generated by using DNAPlotter [42]

\section{Classification of mitochondrial haplogroups in the Korean population}

We examined all haplogroups previously reported in the Korean population in Korean Genome Project (Korea $1 K)(n=1094)$ [23], and listed in the Korean National Standard Reference Variome Database of whole genomes (KoVariome) [43]. Eleven variants (m.73A $>\mathrm{G}$, m.263A > G, m.750A > G, m.1438A $>$ G, m.2706A $>$ G, m.4769A $>$ G, m.7028C $>$ T, m.8860A $>$ G, m.11719G $>A$, $\mathrm{m} .14766 \mathrm{C}>\mathrm{T}$, and $\mathrm{m} \cdot 15326 \mathrm{~A}>\mathrm{G}$ ) with an overall frequency $\geq 50 \%$ that are widespread across all lineages $(\mathrm{L}$, $\mathrm{M}$, and N) [44] were predominant in our study. Among these, five mtDNA variants present at $\geq 80 \%$ in lineages $\mathrm{L}, \mathrm{M}$, or $\mathrm{N}$ were found in all individuals. The rare mutation m.73A $>\mathrm{C}$ was observed in one individual in our population. The most dominant haplogroup for the Korean population was found to be D (34.74\%), followed by $M(16.1 \%)$. These haplogroups are prevalent in Asian populations [45] and were diverse in our study subgroups (Fig. 3A, Table 1). The D2 sub-haplogroup, defined by mutation m.16271T $>\mathrm{C}$ [46] and possibly equating to D4e1b [47], was observed in one subject. Consequently, $87.8 \%$ of haplogroup D belonged to the D4 subgroup, which had the highest frequency in our Korean population; the D5 subgroup was observed in all remaining subjects. These results were consistent with the data of Korea 1K, which includes sequence data from 1094 whole genomes.

The distributions of the haplogroups in the Korean population were obtained from Korea $1 \mathrm{~K}$ [23], KoVariome [43] and five super-populations in the 1000 Genomes Project (1 KG) [33] and then compared to our data. Our study showed the concordance of the haplogroups of the Korean and East Asian populations (1 KG-EAS) (Fig. 3B). Other super-populations had distinct mtDNA haplogroups, as described elsewhere [19]. Haplogroups M, A, $\mathrm{H}$ and $\mathrm{L}$ were most prevalent in South Asian (1 KG-SAS), American (1 KG-AMR), European (1 KG-EUR) and African (1 KG-AFR) populations, respectively.

\section{Annotation of mtDNA variants and drug-induced toxicity}

The D-loop is $1122 \mathrm{bp}$ in length, and consists of two hypervariable regions (HVI at nucleotides 16,024-16,383 and HVII at nucleotides 57-372) and a tandem repeat of poly $(C)$ at nucleotides 303-315 [48]. In all subjects, the mtDNA D-loop region had 74 polymorphisms. All 118 samples had the polymorphism m.263A $>$ G in HVII and one subject carried m.73A $>C$, which was reported to be rare in Asian p opulations (0.01\% in healthy individuals and $0.09 \%$ in patients). In $30 / 118(25.42 \%)$ of the samples 
(A)

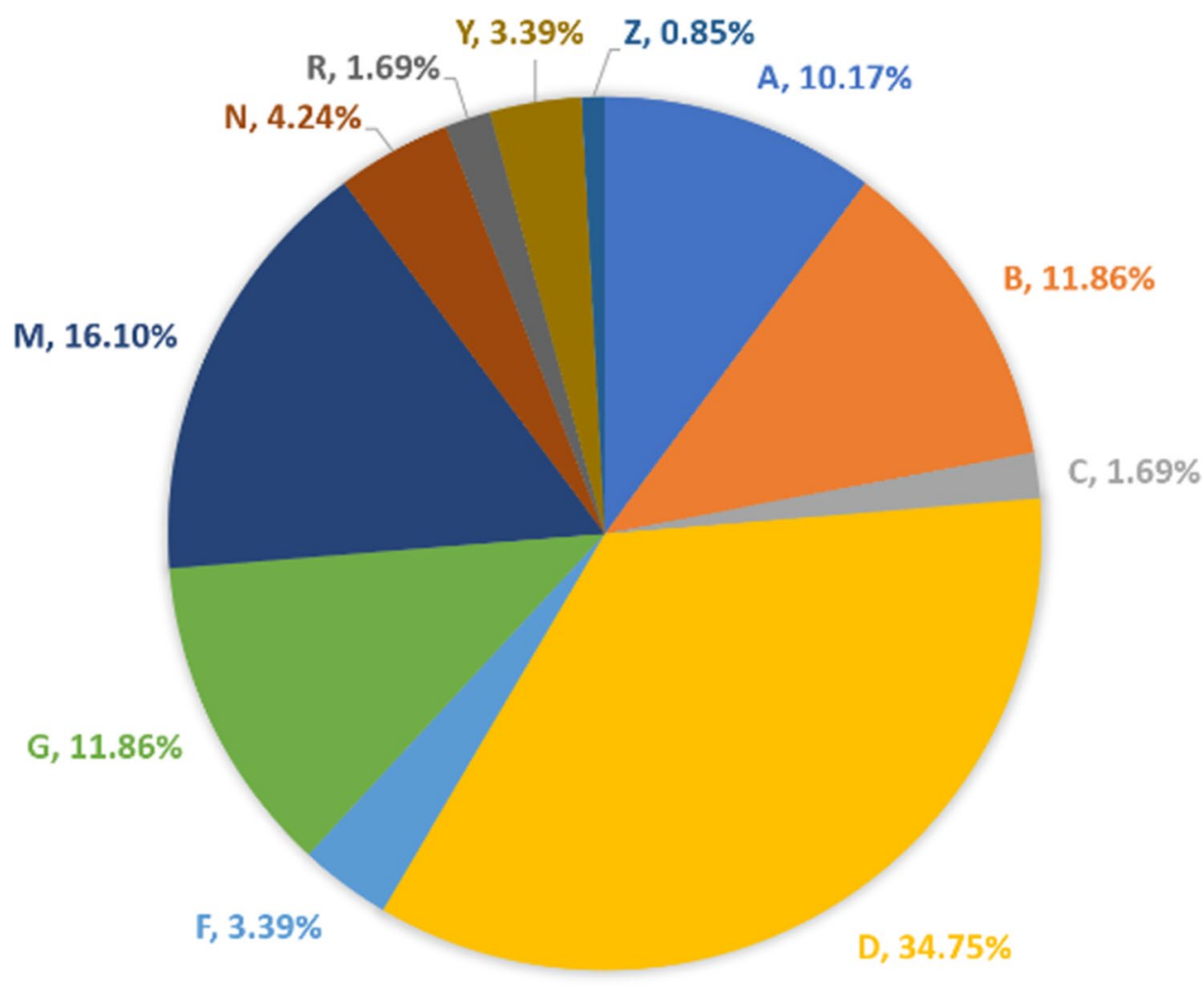

(B)

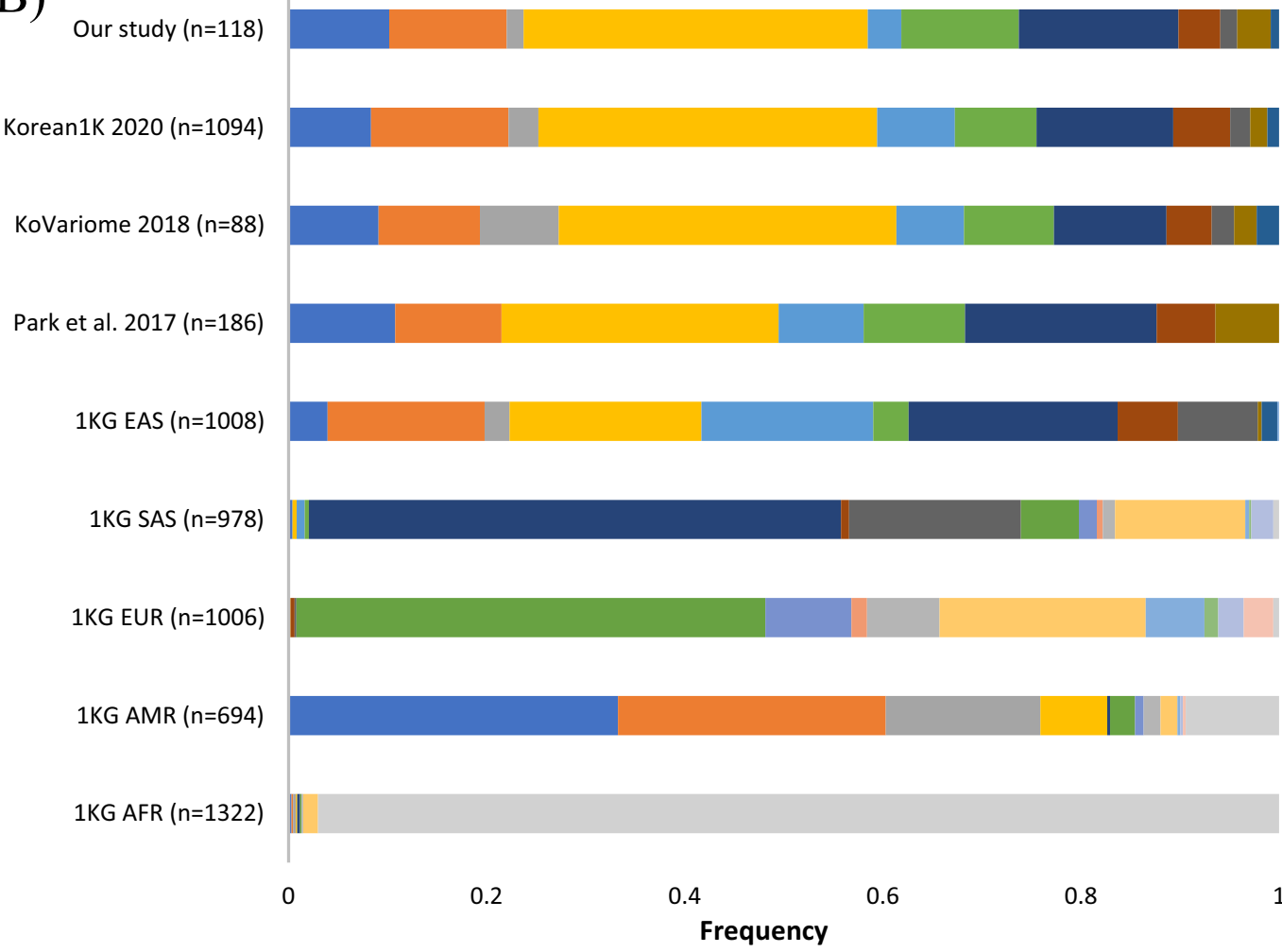

- A

- B

- C

D

- $\mathrm{F}$

G

- $\mathrm{M}$

- N

- $\mathrm{R}$

- $\mathrm{Y}$

- Z

- $\mathrm{H}$

T

$\mathrm{x}$

J

U

K

- I

w

- V

- $\mathrm{L}$

Fig. 3 The haplogroup of mitochondrial genomes for Korean population. A Distribution of haplogroups in the Korean population in the present study. B Stacked bar plots of the frequencies of haplogroups in the Korean population and five super-populations from the 1000 Genomes Project $(1 \mathrm{KG})$ 
Table 1 The sub-haplogroups observed in our study $(n=118)$ based on entire mitochondrial genome variants by using PhyloTree 17 nomenclature in Haplogrep2 analysis

\begin{tabular}{|c|c|c|}
\hline Haplogroup & Sub-haplogroup & $\begin{array}{l}\text { Number of } \\
\text { subjects } \\
(n=118)\end{array}$ \\
\hline A & $A+152+16,362, A 1, A 11, A 5 a, A 5 a 1 a, A 5 b 1 a, A 5 b 1 b, A 6$ & $12(10.17 \%)$ \\
\hline B & B4a1b1a, B4a1c1a1, B4a4, B4b1a2, B4b1a2a, B4c1a1a, B4c1a1b, B4d3a1, B4f1, B5a2a1 + 16,129, B5b, B5b1, B5b2a2 & $14(11.86 \%)$ \\
\hline C & C4a1a, C7a1c, & $2(1.69 \%)$ \\
\hline D & $\begin{array}{l}\text { D2, D4, D4 + 195, D4a, D4a1, D4a1c, D4a1h, D4a3b, D4a3b1, D4b2, D4b2a2a1, D4b2b, D4b2b1, D4c1a, D4c2c, } \\
\text { D4e1a, D4e2, D4f1, D4g1, D4g1a, D4g1b, D4g1c, D4h1a, D4h1c1, D4i, D4n, D5a, D5a2a1 + 16,172, D5b, D5b1, } \\
\text { D5b1b }\end{array}$ & $41(34.75 \%)$ \\
\hline $\mathrm{F}$ & F1a1, F2, F2f, F2i, & $4(3.39 \%)$ \\
\hline G & G1a1, G1a1a, G1a1a1, G2a + 152, G2a1, G2a1 + 16,189+ 16,194, G2a1b, G2a1e, G2a5, G2b2 & $14(11.86 \%)$ \\
\hline M & $\begin{array}{l}\text { M10, M10a1a1b, M10a1b, M11b1a, M7a1a, M7a1a1, M7a1a5, M7b1a1a1, M7c1a2a1, M7c1a3, M7c1a5, M7c1b, } \\
\text { M8a3a, M8a3a1, M9a1a1, M9a1a1a }\end{array}$ & $19(16.1 \%)$ \\
\hline N & $\mathrm{N9a2}$, N9a2a, N9a2c, N9a2d & $5(4.24 \%)$ \\
\hline R & $R+16,189, \mathrm{R} 11 \mathrm{a}$ & $2(1.69 \%)$ \\
\hline Y & Y1 & $4(3.39 \%)$ \\
\hline Z & Z4a & $1(0.85 \%)$ \\
\hline
\end{tabular}

in the present study, a thymine to cytosine $(\mathrm{T}>\mathrm{C})$ transition was found at position 16,189 , producing an uninterrupted homopolymeric tract at mtDNA segment $16,180-16,195$ in HVI. In total, 3 of the 118 samples (2.5\%) had 12 uninterrupted cytosine residues, with the heteroplasmy level of m.16189T > C varying from $54.6 \%$ to $99.9 \%$. Meanwhile, 2 of the 118 (1.7\%) samples had length heteroplasmy in the mitochondrial HVII segment at positions 303-315 due to the insertion of cytosine at position 309, and one subject showed replication slippage in the case of a $\mathrm{T}>\mathrm{C}$ transition at position 310 .

We observed a total of 40 mitochondrial variants in mitochondrially encoded 12S rRNA (MT-RNR1) and mitochondrially encoded $16 \mathrm{~S}$ rRNA (MT-RNR2) genes. Several mtDNA mutations associated with antibioticinduced toxicity were detected in these two genes (Table 2). The m.1555A $>\mathrm{G}$ and m.1494C $>\mathrm{T}$ mutations are well known to be associated with ototoxicity in patients treated with aminoglycoside antibiotics, but were not observed in our population. We may not have been able to detect these rare variants, reported to be found at a rate of 2 per 2922 individuals $(0.07 \%)$ for $\mathrm{m} .1555 \mathrm{~A}>\mathrm{G}$ in the Korean population [49], due to the small sample size. Other aminoglycoside-induced hearing loss variant, m.1189T > C, was observed in $1.69 \%$ of all samples; the rate was previously reported as $0.34 \%$ for the Korean population [50, 51]. Variant at position 961 was identified at a frequency of 5.93\%; these are more likely to be found in Korean than Asian populations (1-3\% in the $1 \mathrm{KG}$ ). The polymorphism of $\mathrm{m} .2706 \mathrm{~A}>\mathrm{G}$ transition, a variation previously suggested to lead to a pred isposition to linezolid-associated lactic acidosis [52], was dominant in all subjects. The m.3010G $>$ A allele, which is associated with linezolid-induced mitochondrial toxicity, was more

Table 2 Frequencies of mitochondrial variants in association with drug-induced toxicity in this study $(n=118)$

\begin{tabular}{|c|c|c|c|c|c|}
\hline Gene & Variant & rsID & Drug & Clinically relevant & $\begin{array}{l}\text { Frequency in } \\
\text { our study (\%) }\end{array}$ \\
\hline \multirow[t]{5}{*}{$M T-R N R 1$} & $m .663 A>G$ & rs56489998 & Aminoglycoside & Ototoxicity [51] & 10.17 \\
\hline & $\mathrm{m} .961 \mathrm{~T}>\mathrm{C}$ & rs3888511 & Aminoglycoside & Ototoxicity [51] & 5.93 \\
\hline & $\mathrm{m} .961 \mathrm{~T}>\mathrm{C}$ & rs3888511 & Linezolid & Mitochondrial toxicity [54] & 5.93 \\
\hline & m.1095T>C & rs267606618 & Aminoglycoside & Ototoxicity [51] & 0.85 \\
\hline & m.1189T >C & rs28358571 & Aminoglycoside & Ototoxicity [51] & 1.69 \\
\hline \multirow[t]{2}{*}{ MT-RNR2 } & $m .2706 A>G$ & rs2854128 & Linezolid & Lactic acidosis, Mitochondrial toxicity [54] & 99.15 \\
\hline & m.3010G > A & rs3928306 & Linezolid & Mitochondrial toxicity [54] & 30.51 \\
\hline MT-ND3 & m.10398A > G & NA & ART & Metabolic/ cardiovascular complications [15] & 70.34 \\
\hline
\end{tabular}


common in the Korean population (30.51\%), consistent with previous studies $[45,53]$. Lastly, we observed a high frequency of m.10398A $>\mathrm{G}$ variant that was reported to influence metabolic ART effects [15] at homoplasmic level in our study.

\section{Discussion}

The D-loop has a regulatory role in mtDNA transcription and replication [55]. Mutations in the D-loop region may significantly affect mtDNA copy number and the gene expression of mitochondrial genomes, thereby disrupting the function of mitochondria, oxidative phosphorylation and ATP production. Here, we found the largest number of polymorphisms in the D-loop region in all subjects. Notably, $25.42 \%$ of the samples harboured the mutation m.16189T $>C$, which is very close to the mtDNA origin of replication and is significantly associated with various non-communicable diseases [56-59]. This could be explained by the lower binding affinity of proteins to the regi on with the $16189 \mathrm{C}$ variant [56]. A negative correlation between continuous cytosines at the mtDNA segment 16,180-16,195 in HVI and mtDNA copy number was reported previously in human peripheral blood cells [60]. The reduction of mitochondrial copy number might lead to the mitochondrial dysfunction that is potentially a mechanism of drug-induced toxicity [61]. It suggested that $\mathrm{m} \cdot 16180 \mathrm{~T}>\mathrm{C}$ variant might increase the risk of developing adverse drug reaction. Thus, the impact of this variant should be considered in further investigation.

The non-synonymous m.10398A $>\mathrm{G}$ polymorphism (overall frequency $=70.34 \%$ ) was reported to be associated with long-term ART-induced toxicity. Notably, most of the variants mentioned above showed homoplasmy (>98.4\% of all mtDNA sequences analysed [62]). The phenotypic expression of pathogenic and heteroplasmic mtDNA mutations could be modulated by homoplasmic mtDNA variants [15]. This suggests that determination of these variants prior to treatment could be useful to identify tuberculosis and HIV patients at risk of drug-induced toxicity.

The frequency of length heteroplasmy in the homopolymeric $\mathrm{C}$-stretch regions was as low as $\sim 4 \%$ in our population, and we detected only one individual with possible length heteroplasmy at position 303-315. Compared to point heteroplasmy, length heteroplasmy is more common and less population-specific [63]. The presence of a tandem poly $(\mathrm{C})$ repeat in the D-loop region of nucleotides $303-315$ and $16,182-16,189$, as a result of polymerase misincorporation or slippage during mtDNA replication [64], reduced the mtDNA copy number. These length heteroplasmies were reportedly associated with a more than twofold increase in cancer risk [65]. In addition, these length variants have been suggested to be associated with several common diseases, including diabetes mellitus and dilated cardiomyopathy [66], and are therefore of clinical importance.

The human mitochondrial genome consists of 13 protein-coding genes, 22 tRNAs and 2 rRNAs. The proteins encoded by the 13 mitochondrial-coding genes are constituents of the enzyme complexes of the oxidative phosphorylation system, enabling mitochondria to act as the cellular powerhouse. They are distributed among various respiratory chain components, in a small part of complex I and throughout complexes III-V (ATP synthase) of the respiratory chain [67].

Mitochondrial rRNA more closely resembles bacterial than human rRNA. However, genetic polymorphisms in these genes can result in greater similarity between mitochondrial and bacterial rRNA, thereby facilitating the binding of anti-microbials (most notably aminoglycosides). In cases of tuberculosis infection treated via multiantibiotic administration, the associations of mtDNA instability and variation with drug-induced toxicity should be taken into consideration. The polymorphisms in the MT-RNR1 and MT-RNR2 genes were associated with increased mitochondrial and clinical adverse effects, most commonly ototoxicity and peripheral neuropathy $[54,68]$. The mutation of mitochondrial 12S rRNA gene is associated with both aminoglycoside-induced deafness and non-syndromic hearing loss. This study detected higher allele frequency in some variants $(\mathrm{m} .663 \mathrm{~A}>\mathrm{G}$, m.961T > C, m.3010G > A) compared to previous studies $[15,46]$. This suggested that these variants should be considered carefully in studies of the Korean population. It has recently been reported that mtDNA polymorphisms may affect respiratory chain function and first-line antituberculosis drug-induced liver injury. However, the underlying mechanisms have yet to be elucidated [69, 70].

The rare mutations observed in our clinical samples may have highly disruptive effects on mitochondrial ribosomes; based on previous study, potential disruption is classified as 'proven' or 'not enough evidence' (NEE) [71, 72]. Among 52 and 145 mutations in the MT-RNR1 and $M T-R N R 2$ genes, respectively, only three $(\mathrm{m} .1494 \mathrm{C}>\mathrm{T}$, m.1555A > G and m.1843T > C) were 'proven' and some of those variants were interpreted in clinical practice for MT-RNR1 gene [51]. Most of the remaining variants were classified as NEE, so it is not yet clear whether changes in mitochondrial ribosomes affect the binding sites of antibiotic drugs to reduce drug toxicity. Other mtDNAencoded ribosome components were suggested to have potential benefits in terms of drug susceptibility and should therefore be investigated further in clinical studies. In the present study, only m.2150 $>\mathrm{A}$, classified as 
NEE, was observed (at a rate of $4.24 \% ; 5 / 118$ samples) as a homoplasmic mutant.

In addition, adverse drug reactions were reported in patients inheriting pathogenic mtDNA mutations. Antibiotic drugs were reported to be associated with optic neuropathy in Leber's hereditary optic neuropathy (LHON) carriers. In patients carrying the m.11778G $>$ A (MT-ND4) mutation, erythromycin has the potential to catalyse a bioenergetic crisis at onset of LHON [73], while ethambutol was suggested to have a synergistic and deleterious effect on tissue specificity, as reviewed elsewhere [74]. Therefore, the use of erythromycin and, by extension, other macrolides, should be avoided in patients with pre-existing pathogenic mtDNA mutations.

The polymorphism of mitochondrial genome has been shown to be associated with $\mathrm{v}$ arious pathophysiological conditions, and could play a role in various physiological and pathological characteristics [3]. The high variability in human mitochondria has been investigated for common diseases [2]. At the time of study, ninety-five mitochondrial markers have been confirmed to be pathogenic [4]. Pathogenic mtDNA mutations were commonly found in a number of major haplogroups in the general population, indicating that many healthy individuals carry these potentially harmful mtDNA mutations. Therefore, mtDNA mutations could serve as biomarkers facilitating early detection and prediction of the prognosis of disease. However, our study identified no pathogenic variants, possibly due to its small sample size.

In European populations, it has been reported that specific mtDNA haplogroups were associated with ARTinduced peripheral neuropathy and metabolic disorders [10-15]. In addition, many studies have demonstrated relationships between haplogroups and cancer risk. However, at the time of our study, there had been no investigations of the relationships between haplogroups and drug-induced toxicity for Korean population. Haplogroups vary widely across ethnic groups, even between South and East Asians. Therefore, the characteristics of haplogroups in specific populations should be considered when exploring the susceptibility of these populations to diseases and drug-induced toxicity.

In this study, we aimed to characterizing the mitochondrial genome profile for Korean population and observed only few known-mutations related to ADRs that could be the explanation for the absence of side effects in 68 subjects who were treated with the first-line antituberculosis drug. However, the characterized variants might contribute to ADRs related to other drugs. For future direction, we choose the subjects to further explore the association of mitochondrial variants with ADRs. In addition to mitochondrial polymorphism, many other factors including nucleus genes, comorbidity, comedication etc. could induce mitochondrial dysfunction that leads to ADRs. Consideration of all these factors will allow us to explore the insight of the association between mtDNA variants and drug-induced toxicity in future study.

\section{Conclusions}

Drug-induced toxicity via mitochondrial dysfunction has been studied extensively. However, the contributions of mitochondrial variants to drug-induced toxicity remain unclear. The present study identified distinct haplotypes in a Korean population, consistent with the results reported previously by Korea $1 \mathrm{~K}$. Despite the relatively small sample size of our study, we investigated annotation between mtDNA characteristics and risk factors for certain diseases and drug-induced toxicity, through NGS analysis. Further studies including larger cohorts are needed to examine the associations of mtDNA with adverse drug reactions in the Korean population. For this issue, we are going to extend the investigation in association with antituberculosis drug-induced toxicity such as drug-induced liver injury and peripheral neuropathy.

\section{Supplementary Information}

The online version contains supplementary material available at https://doi. org/10.1186/s12920-021-01153-0.

Additional file 1. Supplementary material.

\section{Acknowledgements}

We would like to thank to Dr. Nguyen Phuoc Long for his valuable comments for the study. We also grateful to the staffs in Pharmarcogenomic Research Center, Inje University for their supporting.

\section{Authors' contributions}

$\mathrm{PVH}$ involved on study design and experimental conduction, data analysis and manuscript preparation; NVL involved on data analysis and manuscript preparation; $\mathrm{H}$-EJ involved on study design, data analysis, and manuscript editing; Y-SC involved on data analysis and manuscript revision; J-GS involved on project and study design, and data analysis, and manuscript edition and funding of grant. All authors read and approved the final manuscript.

\section{Funding}

This work was supported by the National Research Foundation of Korea (NRF) grant funded by Korean government (MIST) (No. 2018R1A52021242) The funding source had no role in the study design, in the data collection or analysis, in the preparation of the manuscript, or in the decision to submit the paper for publication.

\section{Availability of data and materials}

The datasets generated during the current study are available in the PRJNA774877 repository at this link: https://www.ncbi.nlm.nih.gov/sra/?term= PRJNA774877.

\section{Declarations}

Ethics approval and consent to participate

The study was conducted following the Declaration of Helsinki and institutional criteria. The institutional review boards (IRBs) of all participating sites reviewed and approved the research. Written informed consents were obtained from all participants. 


\section{Consent for publication}

Not applicable.

\section{Competing interests}

The authors state that they have no conflict of interest.

\section{Author details}

1 Department of Pharmacology and Pharmacogenomics Research Center, Inje University, College of Medicine, 633-165 Gaegum-Dong, Jin-Gu, Busan, Republic of Korea. ${ }^{2}$ Department of Precision Medicine, SPMED Co., Ltd., Busan 46508, Republic of Korea. ${ }^{3}$ Department of Pharmacology and Clinical Pharmacology, PharmacoGenomics Research Center, Inje University College of Medicine, Busan 47392, Republic of Korea. ${ }^{4}$ Center for Personalized Precision Medicine of Tuberculosis, Inje University College of Medicine, Busan, Republic of Korea.

Received: 14 October 2021 Accepted: 16 December 2021

Published online: 03 January 2022

\section{References}

1. Wallace DC, Mitochondrial DNA. Variation in human radiation and disease. Cell. 2015;163:33-8.

2. Li H, Slone J, Fei L, Huang T. Mitochondrial DNA variants and common diseases: a mathematical model for the diversity of age-related mtDNA mutations. Cells. 2019;8:608.

3. Tuppen HAL, Blakely EL, Turnbull DM, Taylor RW. Mitochondrial DNA mutations and human disease. Biochim Biophys Acta - Bioenerg. 2010;1797:113-28.

4. ConfirmedMutations < MITOMAP < Foswiki. https://www.mitomap.org/ foswiki/bin/view/MITOMAP/ConfirmedMutations. Accessed 10 Oct 2021.

5. Elliott HR, Samuels DC, Eden JA, Relton CL, Chinnery PF. Pathogenic mitochondrial DNA mutations are common in the general population. Am J Hum Genet. 2008;83:254-60.

6. Boelsterli UA, Lim PLK. Mitochondrial abnormalities-a link to idiosyncratic drug hepatotoxicity? Toxicol Appl Pharmacol. 2007;220:92-107. https://doi.org/10.1016/j.taap.2006.12.013.

7. Penman SL, Carter AS, Chadwick AE. Investigating the importance of individual mitochondrial genotype in susceptibility to drug-induced toxicity. Biochem Soc Trans. 2020;48:787-97. https://doi.org/10.1042/BST20 190233.

8. Yuan Y, Ju YS, Kim Y, Li J, Wang Y, Yoon CJ, et al. Comprehensive molecular characterization of mitochondrial genomes in human cancers. Nat Genet. 2020:52:342-52. https://doi.org/10.1038/s41588-019-0557-x.

9. Zong WX, Rabinowitz JD, White E. Mitochondria and cancer. Mol Cell. 2016:61:667-76. https://doi.org/10.1016/j.molcel.2016.02.011.

10. Hulgan T, Haas DW, Haines JL, Ritchie MD, Robbins GK, Shafer RW, et al. Mitochondrial haplogroups and peripheral neuropathy during antiretroviral therapy: an adult AIDS clinical trials group study. AIDS. 2005;19:13419. https://doi.org/10.1097/01.aids.0000180786.02930.a1.

11. Canter JA, Robbins GK, Selph D, Clifford DB, Kallianpur AR, Shafer R, et al African mitochondrial DNA subhaplogroups and peripheral neuropathy during antiretroviral therapy. J Infect Dis. 2010;201:1703-7. https://doi. org/10.1086/652419.

12. Hendrickson SL, Kingsley LA, Ruiz-Pesini E, Poole JC, Jacobson LP, Palella FJ, et al. Mitochondrial DNA haplogroups influence lipoatrophy after highly active antiretroviral therapy. J Acquir Immune Defic Syndr. 2009;51:111-6. https://doi.org/10.1097/QAl.0b013e3181a324d6.

13. Micheloud D, Berenguer J, Guzmán-Fulgencio M, Campos Y, GarcíaÁlvarez M, Catalán P, et al. European mitochondrial DNA haplogroups and metabolic disorders in HIV/HCV-coinfected patients on highly active antiretroviral therapy. J Acquir Immune Defic Syndr. 2011;58:371-8. https://doi.org/10.1097/QAl.0b013e31822d2629.

14. Hulgan T, Haubrich R, Riddler SA, Tebas P, Ritchie MD, McComsey GA, et al. European mitochondrial DNA haplogroups and metabolic changes during antiretroviral therapy in AIDS Clinical Trials Group Study A5142. AIDS. 2011;25:37-47. https://doi.org/10.1097/QAD.0b013e32833f9d02.

15. Hulgan T, Stein JH, Cotter BR, Murdock DG, Ritchie MD, Dube MP, et al. Mitochondrial DNA variation and changes in adiponectin and endothelial function in HIV-infected adults after antiretroviral therapy initiation. AIDS
Res Hum Retroviruses. 2013;29:1293-9. https://doi.org/10.1089/aid.2013. 0079.

16. Pacheu-Grau D, Gómez-Durá A, López-Pérez MJ, Montoya J, Ruiz-Pesini E. Mitochondrial pharmacogenomics: barcode for antibiotic therapy. Drug Discov Today. 2010. https://doi.org/10.1016/j.drudis.2009.10.008.

17. Stewart JB, Chinnery PF. The dynamics of mitochondrial DNA heteroplasmy: implications for human health and disease. Nat Rev Genet. 2015;16:530-42. https://doi.org/10.1038/nrg3966.

18. Maruyama S, Komuro T, Izawa H, Tsutsumi H. Analysis of human mitochondrial DNA polymorphisms in the japanese population. Biochem Genet. 2013;51:33-70.

19. Yamamoto K, Sakaue S, Matsuda K, Murakami Y, Kamatani Y, Ozono K, et al. Genetic and phenotypic landscape of the mitochondrial genome in the Japanese population. Commun Biol. 2020;3:1-11. https://doi.org/10. 1038/s42003-020-0812-9.

20. Duong NT, Macholdt E, Ton ND, Arias L, Schröder R, Van Phong N, et al. Complete human mtDNA genome sequences from Vietnam and the phylogeography of Mainland Southeast Asia. Sci Rep. 2018;8:11651. https://doi.org/10.1038/s41598-018-29989-0.

21. Stoljarova M, King JL, Takahashi M, Aaspõllu A, Budowle B. Whole mitochondrial genome genetic diversity in an Estonian population sample. Int J Legal Med. 2016;130:67-71.

22. Just RS, Diegoli TM, Saunier JL, Irwin JA, Parsons TJ. Complete mitochondrial genome sequences for 265 African American and U.S. "Hispanic" individuals. Forensic Sci Int Genet. 2008;2:e45-8.

23. Jeon S, Jeon S, Bhak Y, Bhak Y, Bhak Y, Choi Y, et al. Korean Genome Project: 1094 Korean personal genomes with clinical information. Sci Adv. 2020;6:eaaz7835. https://doi.org/10.1126/sciadv.aaz7835.

24. Park S, Cho S, Seo HJ, Lee JH, Kim MY, Lee SD. Entire Mitochondrial DNA Sequencing on Massively Parallel Sequencing for the Korean Population. J Korean Med Sci. 2017;32(4):587. https://doi.org/10.3346/jkms.2017.32.4. 587.

25. Zhou Y, Guo F, Yu J, Liu F, Zhao J, Shen H, et al. Strategies for complete mitochondrial genome sequencing on lon Torrent PGM ${ }^{\top M}$ platform in forensic sciences. Forensic Sci Int Genet. 2016;22:11-21.

26. Gould MP, Bosworth CM, McMahon S, Grandhi S, Grimerg BT, La Framboise T. PCR-free enrichment of mitochondrial DNA from human blood and cell lines for high quality next-generation DNA sequencing. PLoS ONE. 2015;10:1-13.

27. McElhoe JA, Holland MM, Makova KD, Su MSW, Paul IM, Baker CH, et al. Development and assessment of an optimized next-generation DNA sequencing approach for the mtgenome using the Illumina MiSeq. Forensic Sci Int Genet. 2014;13:20-9.

28. Levin BC, Hancock DK, Holland KA, Cheng H, Richie KL. Standard Reference Materials ${ }^{\circledR}$ Human Mitochondrial DNA-Amplification and Sequencing Standard Reference Materials-SRM 2392 and SRM 2392-I.

29. INSTRUCTION MANUAL NEBNext ${ }^{\circledR}$ Ultra $^{\text {TM }}$ II DNA Library Prep Kit for Illumina ${ }^{\circledR}$.

30. Trivedi UH, Cézard T, Bridgett S, Montazam A, Nichols J, Blaxter M, et al. Quality control of next-generation sequencing data without a reference. Front Genet. 2014. https://doi.org/10.3389/fgene.2014.00111.

31. Ewels P, Magnusson M, Lundin S, Käller M. MultiQC: summarize analysis results for multiple tools and samples in a single report. Bioinformatics. 2016;32:3047-8. https://doi.org/10.1093/bioinformatics/btw354.

32. Bolger AM, Lohse M, Usadel B. Trimmomatic: a flexible trimmer for Illumina sequence data. Bioinformatics. 2014;30:2114-20. https://doi.org/10. 1093/bioinformatics/btu170.

33. Auton A, Abecasis GR, Altshuler DM, Durbin RM, Bentley DR, Chakravart $A$, et al. A global reference for human genetic variation. Nature. 2015;526:68-74. https://doi.org/10.1038/nature15393.

34. Li H, Durbin R. Fast and accurate short read alignment with BurrowsWheeler transform. Bioinformatics. 2009;25:1754-60. https://doi.org/10. 1093/bioinformatics/btp324.

35. Okonechnikov K, Conesa A, García-Alcalde F. Qualimap 2: advanced multi-sample quality control for high-throughput sequencing data. Bioinformatics. 2016;32:292-4. https://doi.org/10.1093/bioinformatics/btv566.

36. GATK Best Practices Mitochondrial Analysis_GATK. https://gatk.broad institute.org/hc/en-us/community/posts/360056028651-GATK-Best-Pract ices-Mitochondrial-Analysis. Accessed 5 May 2021.

37. Preste R, Clima R, Attimonelli M. Human mitochondrial variant annotation with HmtNote. bioRxiv. 2019. https://doi.org/10.1101/600619. 
38. Preste R, Vitale O, Clima R, Gasparre G, Attimonelli M. Hmtvar: a new resource for human mitochondrial variations and pathogenicity data. Nucleic Acids Res. 2019;47:D1202-10. https://doi.org/10.1093/nar/gky10 24.

39. Weissensteiner H, Pacher D, Kloss-Brandstätter A, Forer L, Specht G, Bandelt HJ, et al. HaploGrep 2: mitochondrial haplogroup classification in the era of high-throughput sequencing. Nucleic Acids Res. 2016;44:W58-63. https://doi.org/10.1093/nar/gkw233.

40. Kiesler K. Sequencing and standards for characterization of the mitochondrial genome. 2018.

41. Lanave C, Tommasi S, Preparata G, Saccone C. Transition and transversion rate in the evolution of animal mitochondrial DNA. BioSystems. 1986;19:273-83.

42. Carver T, Thomson N, Bleasby A, Berriman M, Parkhill J. DNAPlotter: circular and linear interactive genome visualization. Bioinformatics. 2009;25(1):119-20. https://doi.org/10.1093/bioinformatics/btn578.

43. Kim J, Weber JA, Jho S, Jang J, Jun J, Cho YS, et al. KoVariome: Korean National Standard Reference Variome database of whole genomes with comprehensive SNV, indel, CNV, and SV analyses. Sci Rep. 2018. https:// doi.org/10.1038/s41598-018-23837-x.

44. TopVariants < MITOMAP < Foswiki. https://www.mitomap.org/foswiki/ bin/view/MITOMAP/TopVariants. Accessed 6 May 2021

45. SearchAllele < Main < Foswiki. https://www.mitomap.org/foswiki/bin/ view/Main/SearchAllele. Accessed 6 May 2021.

46. Origin and evolution of Native American mtDNA variation: a reappraisal_PubMed. https://pubmed.ncbi.nlm.nih.gov/8808611/. Accessed 21 June 2021

47. Tanaka M, Cabrera VM, González AM, Larruga JM, Takeyasu T, Fuku N, et al. Mitochondrial genome variation in Eastern Asia and the peopling of Japan. Genome Res. 2004;14(10A):1832-50. https://doi.org/10.1101/gr. 2286304.

48. Mutect2-GATK. https://gatk.broadinstitute.org/hc/en-us/articles/36004 2477952-Mutect2. Accessed 30 April 2021.

49. rs267606617 RefSNP Report-dbSNP—NCBI. https://www.ncbi.nlm.nih gov/snp/rs267606617. Accessed 26 May 2021.

50. rs28358571 RefSNP Report—dbSNP—NCBI. https://www.ncbi.nlm.nih. gov/snp/rs28358571. Accessed 26 May 2021

51. McDermott JH, Wolf J, Hoshitsuki K, Huddart R, Caudle KE, Whirl-Carrillo $M$, et al. Clinical Pharmacogenetics Implementation Consortium (CPIC) guideline for the use of aminoglycosides based on MT-RNR1 genotype. Clin Pharmacol Ther. 2021. https://doi.org/10.1002/cpt.2309.

52. Narita M, Tsuji BT, Yu VL. Linezolid-associated peripheral and optic neuropathy, lactic acidosis, and serotonin syndrome. Pharmacotherapy. 2007;27:1189-97.

53. rs3928306 RefSNP Report-dbSNP_NCBI. https://www.ncbi.nlm.nih gov/snp/rs3928306. Accessed 6 May 2021.

54. Garrabou G, Soriano À, Pinós T, Casanova-Mollà J, Pacheu-Grau D, Morén $C$, et al. Influence of mitochondrial genetics on the mitochondrial toxicity of linezolid in blood cells and skin nerve fibers. Antimicrob Agents Chemother. 2017;61:e00542-17.

55. Greenberg BD, Newbold JE, Sugino A. Intraspecific nucleotide sequence variability surrounding the origin of replication in human mitochondrial DNA. Gene. 1983;21:33-49.

56. Park KS, Chan JC, Chuang LM, Suzuki S, Araki E, Nanjo K, et al. A mitochondrial DNA variant at position 16189 is associated with type 2 diabetes mellitus in Asians. Diabetologia. 2008;51:602-8. https://doi.org/10.1007/ s00125-008-0933-z.

57. Kumar B, Bhat Zl, Bansal S, Saini S, Naseem A, Wahabi K, et al. Association of mitochondrial copy number variation and T16189C polymorphism with colorectal cancer in North Indian population. Tumor Biol. 2017. https://doi.org/10.1177/1010428317740296.

58. Saldaña-Rivera E, Careaga-Castilla MJ, Olvera-Cárdenas GD, Pérez-Soto E Sánchez-Monroy V. Mitochondrial T16189C polymorphism is associated with metabolic syndrome in the Mexican population. Dis Markers. 2018 https://doi.org/10.1155/2018/3981315.

59. Jamali L, Banoei MM, Khalili E, Dadgar S, Houshmand M. Association of genetic variations in the mitochondrial d-loop with $\beta$-thalassemia. Mitochondrial DNA Part A DNA Mapp Seq Anal. 2016;27:1-4. https://doi. org/10.3109/19401736.2014.958730.

60. Liou CW, Lin TK, Chen JB, Tiao MM, Weng SW, Chen SD, et al. Association between a common mitochondrial DNA D-loop polycytosine variant and alteration of mitochondrial copy number in human peripheral blood cells. J Med Genet. 2010;47:723-8. https://doi.org/10.1136/jmg.2010. 077552.

61. Ramachandran A, Visschers RGJ, Duan L, Akakpo JY, Jaeschke H. Mitochondrial dysfunction as a mechanism of drug-induced hepatotoxicity: current understanding and future perspectives. J Clin Transl Res. 2018;4:75. https://doi.org/10.18053/JCTRES.04.201801.005.

62. He Y, Wu J, Dressman DC, lacobuzio-Donahue C, Markowitz SD, Velculescu VE, et al. Heteroplasmic mitochondrial DNA mutations in normal and tumour cells. Nature. 2010;464:610-4. https://doi.org/10.1038/natur e08802.

63. Hauswirth WW, Clayton DA. Length heterogeneity of a conserved displacement-loop sequence in human mitochondrial DNA. Nucleic Acids Res. 1985;13:8093-104. https://doi.org/10.1093/nar/13.22.8093.

64. Howell N, Smejkal CB. Persistent heteroplasmy of a mutation in the human mtDNA control region: hypermutation as an apparent consequence of simple-repeat expansion/contraction. Am J Hum Genet. 2000;66:1589-98. https://doi.org/10.1086/302910.

65. Zhao H, Shen J, Medico L, Platek M, Ambrosone CB. Length heteroplasmies in human mitochondrial DNA control regions and breast cancer risk. Int J Mol Epidemiol Genet. 2010;1:184-92.

66. Avital G, Buchshtav M, Zhidkov I, Tuval J, Dadon S, Rubin E, et al. Mitochondrial DNA heteroplasmy in diabetes and normal adults: role of acquired and inherited mutational patterns in twins. Hum Mol Genet. 2012;21:4214. https://doi.org/10.1093/HMG/DDS245.

67. Anderson S, Bankier AT, Barrell BG, De Bruijn MHL, Coulson AR, Drouin J, et al. Sequence and organization of the human mitochondrial genome. Nature. 1981;290:457-65. https://doi.org/10.1038/290457a0.

68. Nguyen T, Jeyakumar A. Genetic susceptibility to aminoglycoside ototoxicity. Int J Pediatr Otorhinolaryngol. 2019;120:15-9.

69. Mansouri A, Gattolliat CH, Asselah T. Mitochondrial dysfunction and signaling in chronic liver diseases. Gastroenterology. 2018;155:629-47.

70. Lee L-N, Huang C-T, Hsu C-L, Chang H-C, Jan I-S, Liu J-L, et al. Mitochondrial DNA variants in patients with liver injury due to anti-tuberculosis drugs. J Clin Med. 2019;8:1207.

71. Smith PM, Elson JL, Greaves LC, Wortmann SB, Rodenburg RJT, Lightowlers $\mathrm{RN}$, et al. The role of the mitochondrial ribosome in human disease: searching for mutations in 12s mitochondrial rRNA with high disruptive potential. Hum Mol Genet. 2014;23:949-67. https://doi.org/10.1093/hmg/ ddt490.

72. Elson JL, Smith PM, Greaves LC, Lightowlers RN, Chrzanowska-Lightowlers ZMA, Taylor RW, et al. The presence of highly disruptive $16 \mathrm{~S}$ rRNA mutations in clinical samples indicates a wider role for mutations of the mitochondrial ribosome in human disease. Mitochondrion. 2015;25:17-27. https://doi.org/10.1016/j.mito.2015.08.004.

73. Luca CC, Lam BL, Moraes CT. Erythromycin as a potential precipitating agent in the onset of Leber's hereditary optic neuropathy. Mitochondrion. 2004:4:31-6. https://doi.org/10.1016/j.mito.2004.05.002.

74. Yu-Wai-Man P, Griffiths PG, Chinnery PF. Mitochondrial optic neuropathies - disease mechanisms and therapeutic strategies. Prog Retin Eye Res. 2011;30:81-114. https://doi.org/10.1016/j.preteyeres.2010.11.002.

\section{Publisher's Note}

Springer Nature remains neutral with regard to jurisdictional claims in published maps and institutional affiliations. 